\title{
Protect sites and samples
}

\author{
The restrictions and protocols surrounding the collection and storage of field samples in the Earth \\ sciences are not always complied with. Offences must not be taken lightly.
}

Geoscience research typically extends beyond the confines of the university walls. Field work is varied and includes ocean expeditions to study sea creatures living on volcanoes beneath the Pacific Ocean $^{1}$, analysing sunken shorelines in the aftermath of the Haiti earthquake ${ }^{2}$ and measuring carbon storage in the mangrove forests of Borneo ${ }^{3}$. It is a privilege to work in remote and beautiful places that most can only dream of visiting. But with this privilege comes the responsibility to protect the environment for future enjoyment, and to carefully archive and store the hard-won samples.

Sample collection unavoidably includes an element of destruction. As such, it can irreparably damage the environment. Samples should therefore always be taken sensitively - only when absolutely necessary and from discrete locations, avoiding type localities or sites of scientific or didactic interest. A Commentary on page 817 highlights how indiscriminate rock coring has scarred Earth heritage sites in what is tantamount to scientific vandalism.

Adequate regulations are largely in place: in most national parks worldwide, sample collection and even rock hammering is forbidden.

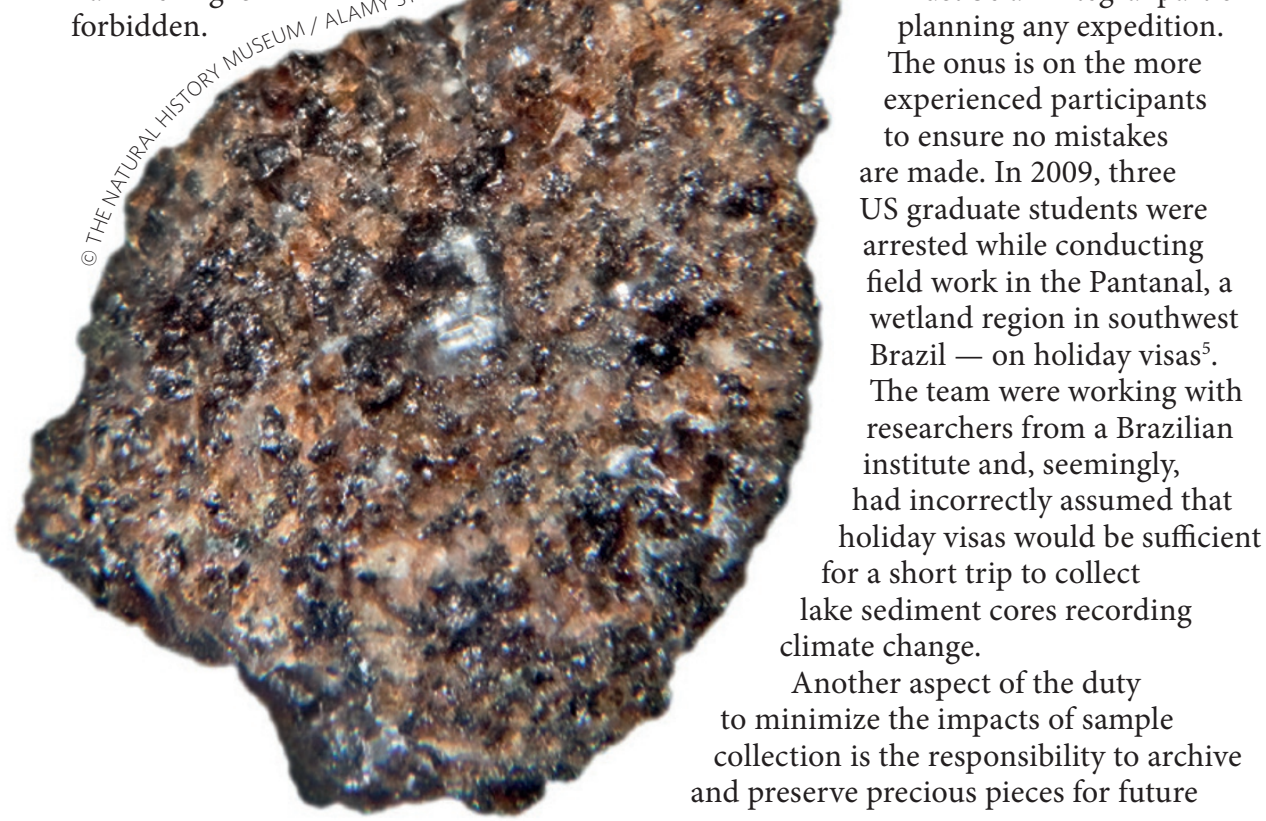

But these rules, both for collection of material and its transport overseas, are often complicated and vary greatly between locations and over time. A prominent case illustrates potential pitfalls. A scientific report describing a four-legged snake that lived about 120 million years ago ${ }^{4}$ was based on a fossil originally found in northeast Brazil, but housed today in a museum in Solnhofen, Germany. A group of palaeontology researchers from the UK say that they stumbled across the fossil there, and went on to study it in detail. A case that may seem innocent enough (after all, the fossil was found in a museum) has now brought on a legal dispute. The export of fossils from Brazil has been illegal since 1942. It is unclear how and when the fossil wound up in a German museum.

Laws restricting sample export exist to help preserve cultural heritage and to develop local scientific expertise, particularly in developing countries. Of course, science may progress faster in a global free-for-all, but considerations of equity and capacity building should take priority.

Taking the time to investigate restrictions on sample collection and transport before embarking on field work must be an integral part of planning any expedition. The onus is on the more experienced participants to ensure no mistakes are made. In 2009, three US graduate students were arrested while conducting field work in the Pantanal, a wetland region in southwest Brazil - on holiday visas ${ }^{5}$. e had incorrectly assumed that holiday visas would be sufficient

limate change.

Another aspect of the dut collection is the responsibility to archive and preserve precious pieces for future The team were working with study. Cupboards bursting with rocks collected by a long-gone PhD student or a professor who has passed away are still far too commonly found in geology departments. But even clearly important samples can go astray. Following the Apollo missions, NASA distributed hundreds of samples of Moon rock and lunar dust across the US states and to several countries as a gesture of goodwill. Many of these samples are now unaccounted for ${ }^{6}$. One rock is thought to lie in a rubbish dump in Dublin - discarded as debris when the observatory housing it burned to the ground in 1977. Today, digital archives, such as iSamples ${ }^{7}$, aim to help connect physical sample collections across the Earth sciences.

The push for responsible sample collection and archiving must come from the whole community, including scientific publishers. We expect authors to adhere to a clear code of ethics, all the way from conception of a project to publication, when they aim to publish in our pages ${ }^{8}$ : we ask authors to provide details of permits in the acknowledgments section. Descriptions of geological samples and specimens must include clear provenance information to ensure full transparency of the research methods. And the samples must be made available for future analyses. Particularly important or rare samples, such as type specimens and meteorites, should be deposited in a recognized museum or collection, where they can be easily accessed and studied by others.

We have to call on our reviewers, to some degree, to help enforce our ethical code of conduct, and we will take any breaches very seriously. Ultimately, it is for the scientific community in the fieldworking disciplines to establish a culture of ethical sample collection.

\footnotetext{
References

1. Nature Geosci. 2, 370 (2009)

2. Nature Geosci. 3, 808 (2010).

3. Nature Geosci. 4, 344 (2011).

4. Martill, D. M., Tischlinger, H. \& Longrich, N. R. Science 349, 416-419 (2015).

5. Stone, A. Accused Americans could be stuck in Brazil for months. USA Today (5 July 2009).

6. Nature 517, 412 (2015)

7. http://earthcube.org/group/isamples

8. http://www.nature.com/authors/policies/availability.html
} 